\title{
Component stimuli, pairing, spatial separation, and identification of a stimulus complex
}

\author{
DONALD L. KING \\ Howard University, Washington, D. C. 20059 \\ and \\ MOEED KHAN \\ Children's Hospital, Washington, D. C. 20009
}

\begin{abstract}
Two halves of Boring's unambiguous figure of an old woman were presented simultaneously (overlapping in time) or successively (not overlapping in time) and positioned so that when they appeared simultaneously the unambiguous figure of an old woman would have been reported. Simultaneous and successive presentations separated by relatively short time intervals increased the likelihood of the subsequently presented ambiguous figure being identified as an old woman relative to a successive presentation separated by a relatively long time interval. Because physical separation of the two halves is analogous to physical separation of eye fixation points, this result, more than previous ones, supports the idea that perseveration of the effects of prior fixations contributes to the object character of perception.
\end{abstract}

What Allport $(1955$, p. 64) called the object character aspect of perception and what can also be referred to as the wholeness, meaning, and identification aspect of perception is obviously an important topic in perception. One problem in understanding the object character nature of visual perception is that fixations occur and are separated by saccadic eye movements. How can the object character of a stimulus complex be apprehended when the retinal stimulation provided by the interaction between the eyes and the stimulus complex occurs in parts separated by time intervals?

The research of Sperling (1960) and others suggests an answer to the stated question. Sperling showed that the neural effects of visual stimuli persisted in time, these effects gradually decreasing until they almost vanished at $.5 \mathrm{sec}$ past stimulus offset. Part of the explanation of the object character nature of visual perception seems to be that the neural effects of prior fixations perseverate to some extent. Simultaneously occurring neural events corresponding to different portions of a stimulus complex that result from perseveration of the effects of just-prior fixations on these different portions would appear to lead to the experience of the stimulus complex as a whole, i.e., to the apprehension of object character.

The Sperling work only suggests that in everyday visual perception the neural effects of successive fixations perseverate and thereby contribute to the occurrence of object character. Further support for

This paper is based on research by Moeed Khan (1972) for which Donald L. King served as principal adviser. Send requests for reprints to Donald L. King, Department of Psychology, Howard University, Washington, D. C. 20059. this idea would be available if it could be suggested that: (a) the neural effect of a component stimulus of a stimulus complex perseverates, (b) this perseverating neural effect combines with the neural effect of a second component stimulus to result in the apprehension of the object character of the stimulus complex, with the object character of the stimulus complex differing from the object characters of the component stimuli, and (c) Points a and b hold when the component stimuli of the stimulus complex are physically separated.

In respect to Point $a$, that the neural effect of a component stimulus perseverates would be analogous to the neural effect of a single fixation perseverating. In respect to Point $b$, maintaining that the object character of a stimulus complex should differ from the object characters of its component stimuli is analogous to thinking of the object character of an object or figure that is looked at as differing from the object characters of the stimuli corresponding to the points of this figure or object that are focused on. (The object characters of the stimuli corresponding to the fixation points are not readily experienced, however, probably at least partly because the neural effects produced by fixations perseverate to help yield the object character of the figure or object looked at.) In respect to Point c, physical separation of component stimuli is analogous to physical separation of fixation points.

Eriksen and Collins $(1967,1968)$ reported findings in accord with Points $a$ and $b$. In this research, the component stimuli of the stimulus complex were superimposed. The present research is similar to that of Eriksen and Collins, but in the present research the component stimuli of the stimulus complex were seen by subjects as occupying different positions in space. 
Thus, the present research relates to Point $c$ as well as to Points $\mathrm{a}$ and $\mathrm{b}$.

\section{METHOD}

Subjects were 72 male and 72 female undergraduates from Howard University.

Pictures, 4.5 in. wide and 6.5 in. high, of Boring's ambiguous figure and Boring's unambiguous figure of an old woman were used. They were obtained by copying and simultaneously enlarging pictures in Figures 6.41 and 6.42 of Kendler (1963). Both figures were cut diagonally in half from top left to bottom right. The left and right halves of each figure will be called $S_{1}$ and $S_{2}$, respectively.

The T2BC tachistoscope and T2BT timer of Gerbrands were used. $S_{1}$ and $S_{2}$ of both figures were mounted on the two surfaces that fitted into the ends of the two arms of the tachistoscope. The surfaces were black. When $S_{1}$ and $S_{2}$ were shown simultaneously, they "fitted together," i.e., normally appearing ambiguous and unambiguous figures were seen with the diagonal cut not being apparent. $S_{1}$ and $S_{2}$ were both approximately 23-5/8 in. from a viewer's eyes. The durations of $S_{1}$ and $S_{2}$ for both the ambiguous and unambiguous figures were always $1.0 \mathrm{sec}$.

Students were recruited from psychology classes. There were nine groups, each consisting of 16 students. Students were assigned randomly to groups, except that $\mathrm{N}$ students were assigned to all groups before $\mathrm{N}+1$ students were assigned to any group.

When a student first came to the experimental room, it was indicated that the purpose of the research was to investigate an aspect of visual perception. The overhead light was then turned off. leaving the room very dimly illuminated.

The experimental procedures divided into training and test phases. In the training phase, six groups received both halves of the unambiguous figure of an old woman. $S_{1}$ preceded $S_{2}$, and the times between the onsets of $S_{1}$ and $S_{2}$ were $0.0, .5,1.0,1.2,1.5$, or $2.0 \mathrm{sec}$. The groups will therefore be called the 0.0 -sec group, the .5-sec group, etc. Because the durations of $S_{1}$ and $S_{2}$ were both 1.0 sec, $S_{1}$ and $S_{2}$ were simultaneous for the 0.0 -sec group, $S_{1}$ and $S_{2}$ overlapped for $.5 \mathrm{sec}$ for the .5-sec group, $S_{2}$ came on the moment $S_{1}$ terminated for the 1.0 -sec group, and $.2, .5$, and $1.0 \mathrm{sec}$ of darkness (blackness) intervened between the offset of $S_{1}$ and the onset of $S_{2}$ for the 1.2-, 1.5-, and 2.0-sec groups, respectively. In addition. an $S_{1}$-only group received only $S_{1}$, an $S_{2}$-only group received only $S_{2}$, and a no-stimulus group received no stimulus. For the $S_{1}$-only, $S_{2}$-only, and no-stimulus groups, the timers were set as for the 1.0-sec group.

Nine training trials were given in blocks of four, one, and four trials. The experimenter and student sat close to the tachistoscope. After the overhead light was turned off. the student was told to look into the eyepiece. Each trial was initiated by the experimenter pressing a switch activating the timers. The timers rotated a full circle for each trial, which took $4.4 \mathrm{sec}$. The experimenter paused for a second after the timers returned to their original positions and then initiated another trial. After the fourth, fifth, and ninth trials, it was indicated that the student should look away from the eyepiece, the overhead light was turned on, and the student was asked to wait outside the room. Two minutes later, the student was admitted back in to the room, the overhead light was turned off, the student was positioned in front of the eyepiece, and the next trial was given. etc. Students were required to leave the room prior to the test phase, because the stimuli in the tachistoscope had to be changed for the test phase. Students were therefore asked to leave the room at the specified times during training to influence them to expect different stimuli would not be presented subsequently.

The test phase consisted of a single trial in which the $S_{1}$ and $S_{2}$ components of the ambiguous figure were presented simultaneously for 1 sec to all groups. The overhead light was then turned on, and the student was asked to describe in writing what he saw in the tachistoscope. The experimenter then read the student's description. If it failed to indicate clearly whether the old woman or young woman was perceived, the experimenter asked the following question: "Regardless of what you saw. does the figure you saw the last time remind you more of an old woman or a young woman?"

\section{RESULTS AND DISCUSSION}

The proportions of students identifying the ambiguous figure as an old woman were $15 / 16$ for the $0.0 \mathrm{sec}, 13,16$ for the $.5 \mathrm{sec}, 10 / 16$ for the $1.0 \mathrm{sec}$, $10 / 16$ for the $1.2 \mathrm{sec}, 9 / 16$ for the $1.5 \mathrm{sec}, 5 / 16$ for the $2.0 \mathrm{sec}, 8 / 16$ for the $S_{1}$-only, $1 / 16$ for the $S_{2}$-only, and $6 / 16$ for the no-stimulus groups. Twenty-five per cent of the written descriptions clearly indicated either an old or a young woman was identified. No disagreements on identifications between the experimenter and a second rater occurred for the written descriptions. The number of subjects given a forced choice between an old and a young woman were $12,10,11,11,10,14,15,14$, and 11 for the 0.0 -sec, .5-sec, 1.0-sec, 1.2-sec, 1.5-sec, 2.0-sec.

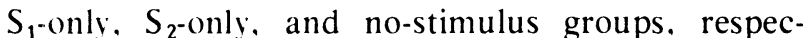
tively, which indicates that differences in proportions identifying the ambiguous figure as an old woman could not have been importantly affected by the method of obtaining this information. The proportions of males and females identifying the ambiguous figure as an old woman did not differ $(\mathrm{p}>.50)$.

Of major interest is the result that the proportion of students identifying the ambiguous figure as an old woman for the 1.0-sec, 1.2-sec, and 1.5-sec groups $[(10+10+9) /(16+16+16)]$ was greater than the corresponding proportion for the 2.0 -sec group, i.e., the group receiving offset of $S_{1}$ of the unambiguous figure separated by onset of $S_{2}$ of the unambiguous figure by the longest time interval $(z=2.02, p<.05)$. Comparing the proportions of identifications of these groups in the way indicated is a priori rather than a posteriori. It was expected that the effect of the first presented component stimulus of the unambiguous figure would decrease as the time interval between the offset of this component stimulus and the onset of the other component stimulus increased. This expectation is supported by the frequently documented result that the neural effects of visual stimuli decrease gradually after stimulus offset.

A result essentially identical to the one just described was also obtained, if it can be assumed that the influences of the $S_{1}$-only and $S_{2}$-only conditions were equivalent, an apparently reasonable but untested assumption. Granted this assumption, the joint effect of being shown both $S_{1}$ and $S_{2}$ of the unambiguous figure when these stimuli are separated by a long time interval should be estimated by the proportion of identitications of the ambiguous figure as an old woman for the $S_{1}$-only and $S_{2}$-only groups $[(1+8) /(16+16)]$. This proportion was less than the proportion of students identifying the ambiguous tigure as an old woman for the 1.0-. 1.2-, and 1.5-sec 
groups $(\mathrm{z}=2.83, \mathrm{p}<.01)$. Thus, two statistical comparisons support the conclusion that when $S_{1}$ and $S_{2}$ of the unambiguous figure did not overlap in time and were separated by relatively brief time intervals, perception of the ambiguous figure was affected in the same direction as when $S_{1}$ and $S_{2}$ of the unambiguous figure overlapped in time.

The results of the previous two paragraphs support Points $\mathrm{a}, \mathrm{b}$, and $\mathrm{c}$ mentioned in the introduction. In respect to Point a, the neural effect of $S_{1}$ of the unambiguous figure should have perseverated for the $1.0-, 1.2-$, and 1.5-sec groups, because the proportion of students in these groups identifying the ambiguous figure as an old woman was greater than both the proportion of students making the same identification in the 2.0-sec group and the proportion of students making the same identification in the $S_{1}$-only and $S_{2}$-only groups. In respect to Point $b$, the perseverating neural effect of $S_{1}$ of the unambiguous figure apparently combined with the effect of $S_{2}$ of the unambiguous figure to result in the apprehension of much the same object character as occurred when $S_{1}$ and $S_{2}$ of the unambiguous figure overlapped in time, because identification of the ambiguous figure as an old woman was greater for both the 0.0 - and .5-sec groups and the 1.0-, 1.2-, and 1.5-sec groups than for the indicated comparison groups. In respect to Point $c, S_{1}$ and $S_{2}$ of the unambiguous figure were separated in space, i.e., were not superimposed. Because Points $a, b$, and $c$ are supported, the idea that simultaneously occurring neural events corresponding to different portions of a stimulus complex and resulting from the effects of just prior fixations perseverating contribute to the apprehension of object character is supported.

\section{REFERENCES}

Allport, F. H. Theories of perception and the concept of structure. New York: Wiley, 1955.

ERIKsen, C. W., \& Collins, J. F. Some temporal characteristics of visual pattern perception. Journal of Experimental Psychology, 1967, 74, 476-484.

Eriksen, C. W., \& Collins, J. F. Sensory traces versus the psychological moment in the temporal organization of form. Journal of Experimental Psychology, 1968, 77, 376-382.

KENDLER, H. H. Basic psychology (2nd ed.). New York: AppletonCentury-Crofts, 1963.

KнаN, M.A study of interstimulus intervals and their effect on the perceptual organization of a figure. Unpublished Master's thesis, Howard University, 1972.

SPERLING, G. The information available in brief visual presentations. Psychological Monographs, 1960, 74, No. 11.

(Received for publication October 5, 1975.) 